\title{
Worsening of Renal Function in Patients with ST- Segment Elevation Myocardial Infarction
}

\author{
Ailed Elena Rodriguez Jiménez* \\ Department of Cardiology, Camilo Cienfuegos Hospital, Cuba \\ *Corresponding author: Ailed Elena Rodriguez Jiménez, Department of Cardiology, Camilo Cienfuegos Hospital, Sancti Spíritus, Cuba

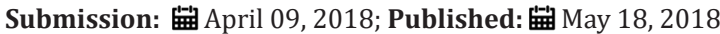

\section{Opinión}

Acute myocardial infarction with ST-segment elevation is a cardiac emergency with high mortality. Patients with ST-segment Elevation Myocardial Infarction (STEMI) should have an early assessment of risk, including an evaluation of renal function, the extent of myocardial damage, the occurrence of successful reperfusion, and the presence of clinical markers of high risk like Killip class $>\mathrm{I}$, anterior myocardial infarction, history of heart failure, or peripheral arterial disease [1].

Despite optimized treatment available for the management of STEMI with primary percutaneous coronary intervention and fibrinolytic agents there is still a high incidence of death and recurrent Acute Myocardial Infarction (AMI) after hospital discharge. The estimated rates vary between $5 \%$ and $10 \%$ within a month after the acute event, and the long-term risk is high [2].

Renal dysfunction [estimated Glomerular Filtration Rate (eGFR) $<30 \mathrm{~mL} / \mathrm{min} / 1.73 \mathrm{~m} 2$ is present in approximately $30-40 \%$ of patients with Acute Coronary Syndrome (ACS) and is associated with a worse prognosis and increased risk of in-hospital complications. However, kidney and heart failure due to AMI are common and frequently co-exist. This interaction is defined as Cardio Renal Syndrome (CRS). A consensus definition and classification about CRS was proposed in 2008 by the Acute Dialysis Quality Initiative work group and identified five CRS sub-types according to disease's onset [3].

The type-1 CRS is characterized by acute worsening of cardiac function leading to kidney disease [3]. Wide spectrum of cardiac conditions that may contribute to a Worsening of Renal Function (WRF) includes acutely de compensated heart failure, acute coronary syndrome, cardiogenic shock, and low-flow syndrome following cardiac surgery. Acute worsening of renal function, is evidenced by an increase higher than $0.3 \mathrm{mg} / \mathrm{dl}$ of baseline serum creatinine, occurring in a variable percentage of $27-40 \%$ of patients with acute coronary syndrome and heart failure [4]. Several potential mechanisms have been proposed to explain the association between renal dysfunction and poor prognosis in ACS patients and have not yet been fully elucidated. In STEMI complicated with heart failure the impairment of blood pressure leads to a series of adjustments that are configured in the decrease of cardiac output, stroke volume, and finally in reduced circulating volume. The critical state and reduced left ventricular ejection fraction are independent predictors of Acute kidney Injury (AKI) in STEMI.

The decrease in arterial circulating blood volume is detected by baroreceptors and causes neurohormonal activation that produces compensatory mechanisms to correct the state of relative hypovolemia and to restore proper tissue perfusion. Subsequently activation of immune system (pro- and anti-inflammatory cytokines and chemokines release) and sympathetic nervous systems, hyperactivity of Renina-Angiotensina-Aldosterone System (RAAS) and coagulation cascade is established. WRF following ACS may possibly serve as a marker of cardiac disease severity [5].

The early detection of WRF after STEMI is important for the decision of treatment options. Hence the identification of risk factors for developing AKI are better to provide individualized the rapeutic options to avoid WRF. Although decisions of reperfusion strategy in patients with STEMI have to be made before serum creatinine is available, it is important to estimate the renal function as soon as possible. The type and dose of antithrombotic agent and the amount of contrast agent could be considered based on renal function. The excess of dose of antithrombotics in patients with WRF, can contribute to an increase of bleeding risk. To guarantee a proper hydration after primary percutaneous coronary intervention and to limit the dose of contrast agents, preferentially low-osmolality contrast agents, are important measures in minimizing the risk of contrast-induced nephropathy.

Cardiorenal syndrome is an independent predictor of in hospital mortality and its detection could be useful in the risk assessment of patients with myocardial infarction. The early identification of risk factors may contribute to reduce the mortality after a complicated acute myocardial infarction in the modern era. This information can be one piece of many to direct clinical decision-making and quality improvement. 


\section{References}

1. Anderson JL, Morrow DA (2017) Acute Myocardial Infarction. N Engl Med 376(21): 2053-2064.

2. Ibanez B, James S, Agewall S, Antunes MJ, Bucciarelli-Ducci C, et al. (2012) ESC Guidelines for the management of acute myocardial infarction in patients presenting with ST-segment elevation. Eur Heart J 33(20): 2569-2619.
3. Ronco C, McCullough P, Anker SD, Anand I, Aspromonte N, et al. (2010) Cardio-renal syndromes: report from the consensus conference of the acute dialysis quality initiative. Eur Heart J 31(6): 703-711.

4. Scabbia EV, Scabbia L (2015) The Cardio-renal Syndrome (CRS). IJC Metabolic \& Endocrine 9: 1-4.

5. Di Lullo L, Bellasi A, Russo D, Cozzolino M, Ronco C (2017) Cardiorenal acute kidney injury: Epidemiology, presentation, causes, pathophysiology and treatment. Int J of Cardiol 227: 143-150.
Creative Commons Attribution 4.0

International License

For possible submissions Click Here

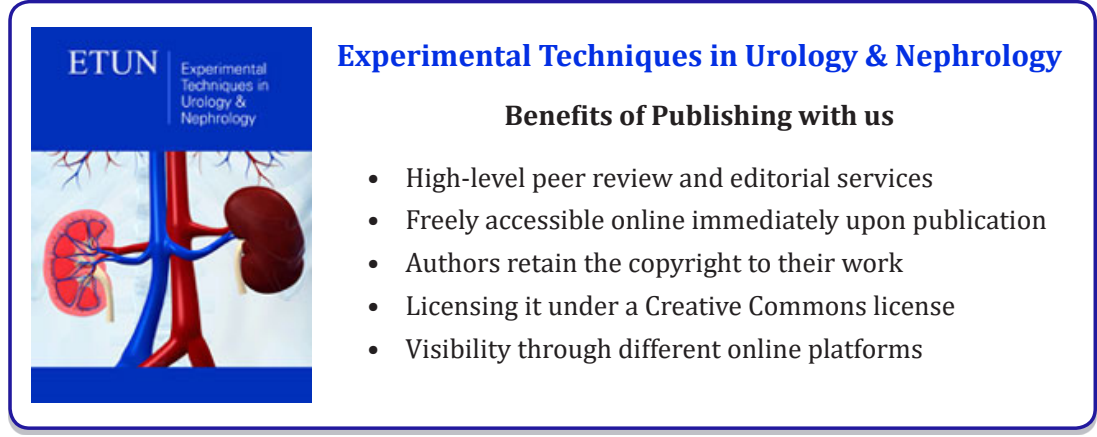

